\title{
Null Controllability of a degenerated reaction-diffusion system in cardiac electro-physiology
}

\author{
Mostafa Bendahmane ${ }^{\mathrm{a}}$ and Felipe Wallison Chaves-Silva ${ }^{\mathrm{b}}$ \\ ${ }^{a}$ Laboratoire de Institut Mathematiques de Bordeaux, Universite Victor Segalen \\ Bordeaux 2, Place de la Victoire, 33076 Bordeaux, France. \\ ${ }^{\mathrm{b}}$ BCAM - Basque Center for Applied Mathematics, Alameda Mazzaredo 14, \\ E-48009 Bilbao - Basque Country - Spain \\ Reçu le ${ }^{* * * * *}$; accepté après révision le +++++ \\ Présenté par £££££
}

\begin{abstract}
This paper is devoted to analyze the null controllability of a nonlinear reactiondiffusion system approximating a parabolic-elliptic system modeling electrical activity in the heart. The uniform, with respect to the degenerating parameter, null controllability of the approximating system by a single control force acting on a subdomain is shown. The proof needs a precisely estimate with respect to the degenerating parameter and it is done combining Carleman estimates and energy inequalities.

To cite this article: M. Bendahmane, F. Chaves, C. R. Acad. Sci. Paris, Ser. I ... (...).

\section{Résumé}

Ce travail est consacré à l'analyse de la nulle contrôlabilité d'un système dégénéré de réaction-diffusion non-linéaire modélisant l'activité électrique du coeur. Notre contrôle agit dans un sous-domaine fixe du domaine du coeur. Nous prouvons la nulle contrôlabilité de notre modèle en établissant en particulier une estimation de Carleman pour l'équation dégénéré. Des estimations globales de type Carleman et la régularité parabolique sont employées.

Pour citer cet article: M. Bendahmane, F. Chaves, C. R. Acad.Sci.Paris, Ser. I $\ldots(\ldots)$.
\end{abstract}

Email addresses: mostafa.bendahmane@u-bordeaux2.fr (Mostafa Bendahmane), chaves@bcamath.org (Felipe Wallison Chaves-Silva). 


\section{Introduction}

Let $\Omega \subset \mathbb{R}^{N}$ be a bounded connected open set whose boundary $\partial \Omega$ is regular enough and $\omega$ a nonempty subset of $\Omega$. Given $T>0$, in $\Omega$ we consider the controllable bidomain model (in $\mathbb{R}^{3}$ it describes electrical activities):

$$
\begin{cases}c_{m} \partial_{t} v-\operatorname{div}\left(\mathbf{M}_{i}(x) \nabla u_{i}\right)+h(v)=f \chi_{\omega} & \text { in } \quad Q:=\Omega \times(0, T), \\ c_{m} \partial_{t} v+\operatorname{div}\left(\mathbf{M}_{e}(x) \nabla u_{e}\right)+h(v)=0 & \text { in } \quad Q:=\Omega \times(0, T), \\ u_{i}=0, \quad u_{e}=0 & \text { on } \quad \Sigma:=\partial \Omega \times(0, T), \\ v(0)=v_{0} & \text { in } \quad \Omega .\end{cases}
$$

In (1), $v=u_{i}-u_{e}, h$ is a nonlinear function, $\chi_{\omega}$ is the characteristic function of $\omega$ and $f$ is a control. We assume that the diffusion matrices $M_{i}$ and $M_{e}$ are of class $C^{\infty}$ and uniformly elliptic in $x$. If $\mathbf{M}_{i} \equiv \mu \mathbf{M}_{e}$ for some constant $\mu \in \mathbb{R}$, then (1) is simplified to the monodomain model

$$
\begin{cases}c_{m} \partial_{t} v-\frac{\mu}{\mu+1} \operatorname{div}\left(\mathbf{M}_{e}(x) \nabla v\right)+h(v)=f \chi_{\omega} & \text { in } Q \\ -\operatorname{div}\left(\mathbf{M}(x) \nabla u_{e}\right)=\operatorname{div}\left(\mathbf{M}_{i}(x) \nabla v\right) & \text { in } Q \\ v=0, u_{e}=0 & \text { on } \Sigma \\ v(0)=v_{0} & \text { in } \Omega\end{cases}
$$

where $\mathbf{M}=\mathbf{M}_{e}+\mathbf{M}_{i}$.

We approximate the monodomain model by the family of parabolic systems

$$
\begin{cases}c_{m} \partial_{t} v^{\epsilon}-\frac{\mu}{\mu+1} \operatorname{div}\left(\mathbf{M}_{e}(x) \nabla v^{\epsilon}\right)+h\left(v^{\epsilon}\right)=f^{\epsilon} \chi_{\omega} & \text { in } Q, \\ \varepsilon \partial_{t} u_{e}^{\epsilon}-\operatorname{div}\left(\mathbf{M}(x) \nabla u_{e}^{\epsilon}\right)=\operatorname{div}\left(\mathbf{M}_{i}(x) \nabla v^{\epsilon}\right) & \text { in } Q, \\ v^{\epsilon}=0, u_{e}^{\epsilon}=0 & \text { on } \Sigma, \\ v^{\epsilon}(0)=v_{0}, u_{e}^{\epsilon}(0)=u_{e, 0} & \text { in } \Omega,\end{cases}
$$

where $\epsilon$ is a positive constant and $u_{e, 0}$ is any function in the space of initial data.

The purpose of this paper is to give an answer to the following question:

If there exists a control $f^{\epsilon}$, for each $\epsilon>0$, that drives the solution $\left(v^{\epsilon}, u_{e}^{\epsilon}\right)$ of (3) to zero at time $t=T$, i.e.

$$
v^{\epsilon}(T)=u_{e}^{\epsilon}(T)=0
$$

is it true that when $\epsilon \rightarrow 0$ the sequence of controls $f^{\epsilon}$ converges to a function $f$ that drives the solution $\left(v, u_{e}\right)$ of (2) to zero at time $t=T$ ? 
Since the bidomain model is a system of two coupled parabolic equations and the monodomain model is a system of parabolic-elliptic type, these two systems have, at least a priori, different control properties. Therefore, it is natural to ask if the controllability of the monodomain model can be seen as a limit process of the controllability of a family of parabolic systems.

It is not difficult to see that a positive answer to this question is equivalent with proving that the control sequence $f^{\epsilon}$ is bounded with respect to $\epsilon$.

In order to answer the question made before, we consider the following linearized version of $(3)$ :

$$
\begin{cases}c_{m} \partial_{t} v^{\epsilon}-\frac{\mu}{\mu+1} \operatorname{div}\left(\mathbf{M}_{e}(x) \nabla v^{\epsilon}\right)+a(x, t) v^{\epsilon}=f^{\epsilon} \chi_{\omega} & \text { in } Q \\ \varepsilon \partial_{t} u_{e}^{\epsilon}-\operatorname{div}\left(\mathbf{M}(x) \nabla u_{e}^{\epsilon}\right)=\operatorname{div}\left(\mathbf{M}_{i}(x) \nabla v^{\epsilon}\right) & \text { in } Q \\ v^{\epsilon}=0, u_{e}^{\epsilon}=0 & \text { on } \Sigma \\ v^{\epsilon}(0)=v_{0}, u_{e}^{\epsilon}(0)=u_{e, 0} & \text { in } \Omega\end{cases}
$$

for a given function $a=a(x, t)$ in $L^{\infty}(Q)$.

Our first main result in this work gives the uniform null controllability of (4).

Theorem 1 Given $v_{0}$ and $u_{e, 0}$ in $L^{2}(\Omega)$, then, for each $\varepsilon>0$, there exists a control $f^{\epsilon} \in L^{2}(\omega \times(0, T))$ so that the solution $\left(v^{\epsilon}, u_{e}^{\epsilon}\right)$ of (4) is driven to zero at time $T$, i.e.

$$
v^{\epsilon}(T)=0, u_{e}^{\epsilon}(T)=0 .
$$

Moreover, the control $f^{\epsilon}$ satisfies

$$
\left\|f^{\epsilon} \chi_{\omega}\right\|_{L^{2}(Q)}^{2} \leq C\left(\left\|v_{0}\right\|_{L^{2}(\Omega)}^{2}+\varepsilon\left\|u_{e, 0}\right\|_{L^{2}(\Omega)}^{2}\right) .
$$

The next second main result gives a positive answer to the question made above.

Theorem 2 Given $v_{0}$ and $u_{e, 0}$ in $L^{2}(\Omega)$ and let $q_{N}$ satisfying

$$
q_{N} \in(2, \infty) \text { if } N=1,2, \frac{N+2}{2}<q_{N}<2 \frac{N+2}{N-2} \text { if } N \geq 3
$$

We have:

- If $h$ is $C^{1}(\mathbb{R})$, global lipschitz and satisfies $h(0)=0$. There exists a control $f^{\epsilon} \in L^{2}(\omega \times(0, T))$ such that the solution $\left(v^{\varepsilon}, u_{e}^{\varepsilon}\right)$ of (3) satisfies

$$
v^{\varepsilon}(T)=u_{e}^{\varepsilon}(T)=0
$$


Besides, the control $f^{\varepsilon}$ has the estimate

$$
\left\|f^{\varepsilon} \chi_{\omega}\right\|_{L^{2}(Q)} \leq C\left(\left\|v_{0}\right\|_{L^{2}(\Omega)}+\varepsilon\left\|u_{e, 0}\right\|_{L^{2}(\Omega)}\right) .
$$

- If $h$ is a $C^{1}$ function satisfying

$$
\begin{gathered}
h(0)=0, \quad \frac{h\left(v_{1}\right)-h\left(v_{2}\right)}{v_{1}-v_{2}} \geq-C, \quad \forall v_{1} \neq v_{2}, \\
0<\liminf _{|v| \rightarrow \infty} \frac{h(v)}{v^{3}} \leq \limsup _{|v| \rightarrow \infty} \frac{h(v)}{v^{3}}<\infty .
\end{gathered}
$$

and $\left(v_{0}, u_{e, 0}\right) \in\left(H_{0}^{1}(\Omega) \cap W^{2\left(1-\frac{1}{q_{N}}\right), q_{N}}(\Omega)\right)^{2}$, with $\left\|\left(v_{0}, u_{e, 0}\right)\right\|_{L^{\infty}(\Omega)} \leq \gamma$, for a sufficient small $\gamma>0$ does not depending on $\varepsilon$. There exists a control $f^{\varepsilon} \in$ $L^{q_{N}}(\omega \times(0, T))$ such that the solution $\left(v^{\varepsilon}, u_{e}^{\varepsilon}\right)$ of $(3)$, with $\left(v^{\varepsilon}, u_{e}^{\varepsilon}\right) \in\left(W_{q_{N}}^{2,1}(Q)\right)^{2}$, satisfies

$$
v^{\varepsilon}(T)=u_{e}^{\varepsilon}(T)=0 .
$$

Moreover, the control $f^{\epsilon}$ has the estimate

$$
\left\|f^{\epsilon} \chi_{\omega}\right\|_{L^{q_{N}}(Q)}^{2} \leq C\left(\left\|v_{0}\right\|_{L^{2}(\Omega)}^{2}+\epsilon\left\|u_{e, 0}\right\|_{L^{2}(\Omega)}^{2}\right) .
$$

Proof of Theorem 2 is done combining Theorem 1 and an appropriate version of Kakutani's fixed point theorem and will not be reproduced here. In the next section we will focus in the proof of Theorem 1 . The detailed proofs will be given in a forthcoming paper.

\section{Uniform null controllability for the linearized system}

It is well known that the proof of Theorem 1 is equivalent with proving an observability inequality for the adjoint system:

$$
\begin{cases}-c_{m} \partial_{t} \varphi^{\epsilon}-\frac{\mu}{\mu+1} \operatorname{div}\left(M_{e}(x) \nabla \varphi^{\epsilon}\right)+a(t, x) \varphi^{\epsilon}=\operatorname{div}\left(M_{i}(x) \nabla \varphi_{e}^{\epsilon}\right) & \text { in } Q, \\ -\varepsilon \partial_{t} \varphi_{e}^{\epsilon}-\operatorname{div}\left(M(x) \nabla \varphi_{e}^{\epsilon}\right)=0 & \text { in } Q, \\ \varphi^{\epsilon}=0, \varphi_{e}^{\epsilon}=0 & \text { on } \Sigma, \\ \varphi^{\epsilon}(T)=\varphi_{T}, \varphi_{e}^{\epsilon}(T)=\varphi_{e, T} & \text { in } \Omega .\end{cases}
$$

More precisely, in order to prove Theorem 1, it is sufficient to show the existence of an universal constant $C$, which is bounded with respect to $\epsilon$, so that the observability inequality

$$
\varepsilon\left\|\varphi_{e}^{\epsilon}(0)\right\|_{L^{2}(\Omega)}^{2}+\left\|\varphi^{\epsilon}(0)\right\|_{L^{2}(\Omega)}^{2} \leq C \iint_{\omega \times(0, T)}\left|\varphi^{\epsilon}\right|^{2} d x d t
$$


holds for every solution of the adjoint system (11) with initial data $\left(\varphi_{T}, \varphi_{e, T}\right) \in$ $L^{2}(\Omega)^{2}$.

To prove (12), we consider $\varphi_{T}$ and $\varphi_{e, T}$ smooth enough and define $\rho^{\epsilon}(x, t)=$ $\operatorname{div}\left(M(x) \nabla \varphi_{e}^{\epsilon}(x, t)\right)$. The pair $\left(\varphi^{\epsilon}, \rho^{\epsilon}\right)$ satisfies:

$$
\begin{cases}-c_{m} \partial_{t} \varphi^{\epsilon}-\frac{\mu}{\mu+1} \operatorname{div}\left(M_{e}(x) \nabla \varphi^{\epsilon}\right)+a(x, t) \varphi^{\epsilon}=\frac{\mu}{\mu+1} \rho^{\epsilon} & \text { in } Q, \\ -\varepsilon \partial_{t} \rho^{\epsilon}-\operatorname{div}\left(M(x) \nabla \rho^{\epsilon}\right)=0 & \text { in } Q, \\ \varphi^{\epsilon}=0, \quad \rho^{\epsilon}=0 & \text { on } \Sigma, \\ \varphi^{\epsilon}(T)=\varphi_{T}, \quad \rho^{\epsilon}(T)=\rho_{T} & \text { in } \Omega .\end{cases}
$$

We apply a Carleman estimate for non degenerate heat equations to equation $(13)_{1}$ and apply a sharp Carleman inequality, with respect to $\epsilon$ (the proof can be found in [1]), to equation $(13)_{2}$. Combining this two inequalities we are able to obtain a Carleman type estimate in the form

$$
\iint_{Q} \beta_{1}^{2}\left|\varphi^{\epsilon}\right|^{2} d x d t+\iint_{Q} \beta_{2}^{2}\left|\rho^{\epsilon}\right|^{2} d x d t \leq C \varepsilon^{-2} \iint_{\omega \times(0, T)} \beta_{3}^{2}|\varphi|^{2} d x d t
$$

for some appropriate weight functions $\beta_{i}:=\beta_{i}(x, t)$ (for $i=1,2,3$ ) and some constant $C=C\left(\Omega, \omega,\|a\|_{\infty}, T\right)>0$.

Next, we get rid of the term $\epsilon^{-2}$ appearing in the right hand side of (14). For that, we take a weight function $\beta_{4}=\beta_{4}(t)$ satisfying $\left|\left(\beta_{4}\right)_{t}(t)\right| \leq C \beta_{2}(x, t)$ for all $(x, t) \in Q$ and we show that

$$
\iint_{Q} \beta_{4}^{2}\left|\rho^{\epsilon}\right|^{2} d x d t \leq C \varepsilon^{2} \iint_{Q} \beta_{2}^{2}\left|\rho^{\epsilon}\right|^{2} d x d t
$$

Inequality (15) is proved applying an energy inequality for the heat like equation satisfied by $\beta_{4} \rho^{\epsilon}$.

We combine (14) and (15) in order to get

$$
\iint_{Q} \beta_{4}^{2}\left|\rho^{\epsilon}\right|^{2} d x d t \leq C \iint_{\omega \times(0, T)} \beta_{3}^{2}\left|\varphi^{\epsilon}\right|^{2} d x d t .
$$

Using (16) and a Carleman estimate to $(13)_{1}$ we show that

$$
\iint_{Q} \beta_{5}^{2}\left|\varphi^{\epsilon}\right|^{2} d x d t \leq C \iint_{\omega \times(0, T)} \beta_{3}^{2}\left|\varphi^{\epsilon}\right|^{2} d x d t
$$

for some appropriate weight function $\beta_{5}:=\beta_{5}(x, t)$. 
Putting (16) and (17) together, we obtain

$$
\iint_{Q} \beta_{5}^{2}\left|\varphi^{\epsilon}\right|^{2} d x d t+\iint_{Q} \beta_{4}^{2}\left|\rho^{\epsilon}\right|^{2} d x d t \leq C \iint_{\omega \times(0, T)} \beta_{3}^{2}|\varphi|^{2} d x d t
$$

Using (18) and energy estimates, it is not difficult to show that

$$
\left\|\varphi^{\epsilon}(0)\right\|_{L^{2}(\Omega)}^{2}+\epsilon\left\|\rho^{\epsilon}(0)\right\|_{L^{2}(\Omega)}^{2} \leq C \iint_{\omega \times(0, T)} \beta_{3}\left|\varphi^{\epsilon}\right|^{2} d x d t
$$

for some constant $C=C\left(\Omega, \omega,\|a\|_{\infty}, T\right)>0$ and it follows from the definition of $\rho^{\epsilon}$ that

$$
\left\|\varphi^{\epsilon}(0)\right\|_{L^{2}(\Omega)}^{2}+\epsilon\left\|\varphi_{e}^{\epsilon}(0)\right\|_{L^{2}(\Omega)}^{2} \leq C \iint_{\omega \times(0, T)}\left|\varphi^{\epsilon}\right|^{2} d x d t
$$

which is the observabilty inequality (12) in the case where we have smooth solutions.

From the density of smooth solutions in the space where the solutions of (11) live, we conclude that the observability inequality is satisfied by all solutions of (11). This concludes the proof of Theorem 1 .

\section{Acknowledgments}

This paper has been partially established during the visit of M. Bendahmane at the Basque Center for Applied Mathematics. The authors thank Professor Enrique Zuazua for various fruitful discussions about this paper. F. Chaves has been partially supported by Grant MTM2008- 03541 of the MICINN, Spain, the ERC Advanced Grant FP7-246775 NUMERIWAVES, ESF Research Networking Programme OPTPDE and the Grant PI2010-04 of the Basque Government.

\section{References}

[1] M. Bendahmane and F.W. Chaves-Silva. Controllability of a degenerate reaction-diffusion system in electrocardiology. Preprint.

[2] E. Fernandez-Cara and E. Zuazua. Null and approximate controllability for weakly blowing up semilinear heat equations. Ann. Inst. Henri Poincare, Analise non lineaire, 17(5), 583-616,2000.

[3] A.V.Fursikov and O.Yu.Imanuvilov. Controllability of Evolution Equations, Lecture Notes Series, Seoul National University, RIM, Global Analysis Research Center, Seoul, 1996. 
[4] O. Glass. A complex-analytic approach to the problem of uniform controllability of a transport equation in the vanishing viscosity limit. J. Funct. Analysis, 358(3): 852-868, 2010.

[5] I. L. Glicksberg. A further Generalization of the Kakutani fixed point theorem, with applications to Nash equilibrium points. Proc. of the $A M S$, serie 3 (9), 170-174, 1952.

[6] S. Guerrero. Null Controllability of some systems of two parabolic equations with one control force. SIAM J. Control and Optimization, 46 (2): 379-394, 2007.

[7] S. Guerrero and G. Lebeau. Singular Optimal Control for a Transport-Diffusion equation. Comm. Partial Diff. Equations, 32: 1813-1836, 2007.

[8] F.A. Khodja, A. Benabdallah, and C. Dupaix. Null-controllability of some reaction-diffusion systems with one control force. J. Math. Anal. Appl., 320: 928-943, 2006.

[9] A. Lopez, X. Zhang and E. Zuazua. Null Controllability of the heat equation as singular limit of the exact controllability of dissipative wave equations. $J$. Math. Pures Appl., 79 (8): 741-808, 2000.

[10] C.-H. Luo and Y. Rudy. A model of the ventricular cardiac action potential. Depolarization, repolarization, and their interaction. Circ Res., 68(6): 15011526, 1991. 